\title{
Modelação MATEMÁtica MACROSCóPICA dA PERdA de CARGa E DA REMOÇÃO DE SÓLIDOS SUSPENSOS TOTAIS NA FILTRAÇÃO DIRETA ASCENDENTE
}

\section{MACROSCOPIC MATHEMATICAL MODELING OF THE HEAD LOSS AND TOTAL SUSPENDED SOLIDS REMOVAL IN UP FLOW DIRECT FILTRATION}

\begin{abstract}
ALEXANDRE BOTARI
Engenheiro Civil. Licenciando em Ciências Exatas com ênfase em Matemática pelo IFSC/USP e Doutor em Engenharia Civil - Área de Concentração: Hidráulica e Saneamento. Escola de Engenharia de São Carlos - USP

\section{LUIZ DI BERNARDO}

Engenheiro Civil. Doutor em Engenharia Civil da Escola de Engenharia de São Carlos - USP. Professor Titular do Departamento de Hidráulica e Saneamento. Escola de Engenharia de São Carlos - USP
\end{abstract}

Recebido: 28/06/06

Aceito: 27/02/07

\section{RESUMO}

A utilização de um modelo matemático permite a obtenção de condiçôes otimizadas de operação, o que conduz à melhoria do controle e da previsão do processo de filtração. Os modelos matemáticos descrevem o processo de filtração através dos mecanismos de remoção de partículas que ocorre no meio filtrante. Este trabalho propõe a modelação matemática baseada nos modelos que descrevem macroscopicamente o processo de filtração e que incluem, por exemplo, o mecanismo de desprendimento de partículas do meio filtrante e a alteração da porosidade do material retido durante a filtração. $\mathrm{O}$ modelo desenvolvido foi adaptado para a aplicação à filtração direta ascendente em material granular de areia grossa inserido no processo de dupla filtração.

\section{ABSTRACT}

The use of a mathematical model allows optimization of the operation conditions, what drives to the improvement of the control and predicting of the filtration process. The mathematical models describe the filtration process through the mechanisms of removal of particles that it happens in the porous media. This work proposes the mathematical modeling based on the models that describe macroscopically the filtration process and that they include, for example, the mechanism of particles detachment from porous media and the alteration of the porosity of retained particles during the filtration. The model developed out adapted for the application to the up flow direct filtration in material to granulate of thick sand inserted in the process of double filtration.

PALAVRAS-CHAVE: Modelo matemático, tratamento de água, dupla filtração, filtração ascendente.

\section{INTRODUÇÃO}

A dupla filtração é composta de duas unidades de filtração associadas para compor uma instalação que consegue somar as características particulares de cada uma delas: a filtração direta ascendente e a filtração descendente. $\mathrm{O}$ filtro ascendente é utilizado na primeira etapa do sistema de dupla filtração e posteriormente seu efluente é encaminhado ao filtro descendente.

A tecnologia da Dupla Filtração (DF), que se caracteriza pela associação em série da Filtração Direta Ascendente e da Filtração Rápida Descendente (FRD), tem as seguintes principais características (Di Bernardo, 2003):

- Maiores custos de implementação, operação e manutenção que a
Filtração Direta Ascendente (FDA), mas menores quando comparados à tecnologia convencional (ciclo completo);

- Possibilidade de tratamento de água bruta de pior qualidade relativamente a FDA;

- Permite o uso de taxas de filtração mais elevadas;

- Atenuação de picos ou variações da qualidade da água bruta em virtude da associação com o filtro descendente que caracteriza esta tecnologia;

- Redução do descarte da água de início de carreira oriunda do filtro ascendente nas FDA.

Esses fatores tornam a tecnologia da dupla filtração mais econômica, proporcionando redução no tamanho da estação de tratamento e sua opera- ção mais simplificada, o que faz com que este tipo de tecnologia seja uma alternativa atrativa.

O objetivo deste trabalho é propor a utilização de um modelo matemático macroscópico semiempírico para a descrição do comportamento da filtração ascendente em leito de multicamadas não uniformes associado a um processo de dupla filtração em meio filtrante de areia grossa.

Vigneswaran \& Chang (1986) desenvolveram um modelo para a filtração descendente que inclui o fenômeno do desprendimento de partículas que ocorre simultaneamente ao fenômeno da agregação durante o processo de filtração. Portanto este modelo pode ser utilizado para descrever todo o ciclo ou "carreira" de filtração e não apenas 
o estágio de maturação e o de serviço, como ocorre nos modelos clássicos.

Neste trabalho, adaptou-se o modelo de filtração de escoamento descendente para o escoamento ascendente, ao passo que o modelo contempla a gradação granulométrica do leito filtrante que é disposto em multicamadas de diferentes faixas granulométricas, além de incluir a variação da porosidade do depósito ao longo da carreira de filtração.

\section{Modelo matemático da filtração}

Para uma dimensão (direção $z$ ), o balanço de massa pode ser escrito, pela abordagem de O’Melia \& Ali (1978), conforme a Equação (1):

$\mathrm{U} \frac{2 \mathrm{n}(\mathrm{z}, \mathrm{t})}{2 \mathrm{z}}+\frac{2 \mathrm{~N}_{\mathrm{A}}(\mathrm{z}, \mathrm{t})}{2 \mathrm{t}}=0$

onde: $U$ é a velocidade ou taxa de filtração $\left(\mathrm{m} . \mathrm{s}^{-1}\right) ; n$ é a concentração ou o número de partículas por unidade de volume de leito $\left(\mathrm{kg}\right.$. $\left.\mathrm{L}^{-1}\right)$ e $N_{A}$ é o número de partículas ou a massa retida por unidade de volume de leito $\left(\mathrm{kg} . \mathrm{L}^{-1}\right)$.

Se for acrescentado o conceito do coeficiente de filtração $(\lambda)$, a equação cinética pode, então, expressar a variação da concentração ou o número de partículas por unidade de volume no espaço e no tempo, conforme a Equação (2):

$\frac{2 \mathrm{~N}_{\mathrm{A}}(\mathrm{z}, \mathrm{t})}{2 \mathrm{t}}=\mathrm{m}(\mathrm{z}, \mathrm{t}) \mathrm{Un}(\mathrm{z}, \mathrm{t})$

O coeficiente do filtro $\lambda(\mathrm{z}, \mathrm{t})$ do modelo de Yao et al (1971), pode ser relacionado à eficiência inicial do coletor (esfera isolada) $\eta(z, t)$, conforme equação (3):

$\mathrm{m}(\mathrm{z}, \mathrm{t})=\frac{3}{2} \frac{\left(1-\mathrm{f}_{0}\right)}{\mathrm{d}_{\mathrm{c}}} \mathrm{h}(\mathrm{z}, \mathrm{t})$

onde $f_{0}$ é a porosidade inicial e $d_{c}$ é o diâmetro do coletor.

Substituindo-se as equaçōes (1) e (2) na eq. (3), tem-se o balanço de massa expresso na forma da Equação (4):

$$
f=\frac{1}{\left(1+10^{(p-x)}\right.}
$$

Para O’Melia \& Ali (1978) a eficiência total do coletor $(\eta)$ pode ser expressa conforme Equação (5):

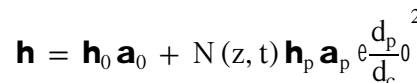

onde: $N(z, t)$ é o número de coletores associados ao grão coletor, que inclui as partículas que funcionam como coletores adicionais, $\eta_{o}$ é a eficiência inicial do coletor; $\eta_{p}$ é a eficiência das partículas que atuam como coletores e $\alpha_{0}$ e $\alpha_{p}$ são o coeficiente de aderência inicial e das partículas aderidas, respectivamente, ao passo que $d_{p}$ e $d_{c}$ são o diâmetro da partícula coletada e o diâmetro do coletor $(\mathrm{m})$, respectivamente.

Entretanto, apenas uma fração $(\beta)$ das partículas aderidas ao coletor atuará efetivamente como coletores adicionais, portanto, a Equação (6) deve ser reescrita na forma:

$h=h_{0} a_{0}+b N_{L}(z, t) h_{p} a_{p} e \frac{d_{p}}{d_{c}}{ }^{2}$

onde $\mathrm{N}_{\mathrm{L}}$ é o número total de partículas removidas pelo coletor limpo (leito limpo) $\left(\mathrm{N}=\beta \mathrm{N}_{\mathrm{L}}\right)$.

Pela definição de eficiência de um único coletor em um escoamento exclusivamente convectivo, obtem-se a Equação (7) para a variação no tempo do número de partículas removidas pelo coletor em leito limpo $\left(\mathrm{N}_{\mathrm{L}}\right)$ e a Equação (8) para a taxa de variação do número total de partículas retidas no coletor $\left(\mathrm{N}_{\mathrm{p}}\right)$ :

$\frac{2 \mathrm{~N}_{\mathrm{L}}(\mathrm{z}, \mathrm{t})}{\mathrm{z}}=\mathrm{h}_{0} \mathrm{a}_{0} \frac{\mathrm{r}}{4} \mathrm{~d}_{\mathrm{c}}^{2} \mathrm{Un}(\mathrm{z}, \mathrm{t})$

$\frac{2 N_{p}}{2 t}=h(z, t) \frac{r}{4} d_{c}^{2} \operatorname{Un}(z, t)$

Para contar com a redução na eficiência de agregação das partículas durante o ciclo de filtração, Vigneswaran \& Chang (1986) adicionaram o termo de desprendimento de partículas aderidas, que segundo os autores, é proporcional ao gradiente hidráulico e ao número de partículas retidas no coletor $\left(\mathrm{N}_{\mathrm{p}}\right)$, ou seja: $\beta_{2} J(z, t) N_{p}(z, t)$. Neste caso, $\beta_{2}$ é a fração de partículas que serão desprendidas, expressa em $\mathrm{s}^{-1}$.

Portanto, o balanço geral será: Número de partículas retidas no coletor $(z, t)=$ Número de partículas retidas no coletor em $t_{0}$ (leito limpo) + Número de partículas retidas por outros coletores (partículas que funcionam como coletores adicionais) - Número de partículas desprendidas. O balanço pode ser expresso na seguinte equação:

$h(z, t) U \frac{r}{4} d_{c}^{2} n(z, t)=h_{0} a_{0} U \frac{r}{4} d_{c}^{2} n(z, t)+$ $+N_{L}(z, t) b a_{p} h_{p} U \frac{r}{4} d_{p}^{2} n(z, t)-b_{2} J(z, t) N_{p}(z, t)$

Isolando-se a eficiência do coletor $(\eta)$, tem-se:

$h(z, t)=h_{0} a_{0}+N_{L}(z, t) b h_{p} a_{p} e{\frac{d_{p}}{d_{c}}}^{2}-$

$-b_{2} J(z, t) \frac{N_{p}(z, t)}{U \frac{r}{4} d_{c}^{2} n(z, t)}$
Os valores $\eta_{0} \alpha_{0}$ e $\eta_{p} \alpha$ são adimensionais. $\mathrm{O}$ número total de partículas retidas pelo coletor ou grão $\left(\mathrm{N}_{\mathrm{p}}\right)$, se relaciona com o número de partículas retidas por unidade de volume de leito filtrante $\left(\mathrm{N}_{\mathrm{b}}\right)$ pela relação $\mathrm{N}_{\mathrm{p}} \mathrm{N}_{\mathrm{c}}=\mathrm{N}_{\mathrm{b}}$. A Equação (11) mostra o número de coletores por unidade de volume $\left(\mathrm{N}_{\mathrm{c}}\right)$ :

$\mathrm{N}_{\mathrm{c}}=>\frac{6\left(1-\mathrm{f}_{0}\right)}{\mathrm{rd}_{\mathrm{c}}^{3}} \mathrm{~F}$

As equações ora descritas contém aproximaçōes para situações ideais, como por exemplo, porosidade constante e partículas e coletores esféricos. A seguir, serão discutidas algumas alteraçôes feitas no modelo básico.

\section{Variação na porosidade}

A variação na porosidade parte do pressuposto de que a deposição de partículas nos vazios intergranulares do leito filtrante modifica suas propriedades ao longo do tempo (massa, massa específica, área superficial e forma). Boller \& Kavanaugh (1995) utilizaram uma interessante analogia entre a floculação e a retenção no coletor do meio filtrante, sugerindo que a retenção de partículas no leito diminui a porosidade ao longo do tempo. A relação mostrada na Equação (12) apresenta esta variação da porosidade durante a retenção de partículas no ciclo de filtração:

$1-\mathrm{f}(\mathrm{z}, \mathrm{t})=\mathrm{N}_{\mathrm{c}} \frac{\mathrm{r}}{6} \mathrm{~d}_{\mathrm{c}}^{3}+\mathrm{N}_{\mathrm{b}} \frac{\mathrm{r}}{6} \uparrow \frac{\mathrm{d}_{\mathrm{p}}^{3}}{1-\mathrm{f}_{\mathrm{d}}} \mathrm{P}$

Considerando-se $\mathrm{N}_{\mathrm{b}}=\mathrm{N}_{\mathrm{c}} \mathrm{N}_{\mathrm{p}}$ e $1-\mathrm{f}_{0}=\mathrm{N}_{\mathrm{c}} \frac{\mathrm{r}}{6} \mathrm{~d}_{\mathrm{c}}^{3}$, a equação (12) se

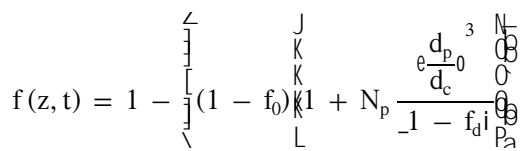

onde $f_{d}$ é a porosidade do depósito ou porosidade dos sólidos retidos no leito filtrante, e tem sido freqüentemente adotada como sendo 0,7 .

O fator $1-f_{0}$ que aparece no numerador representa um fator de correção entre a área específica e o volume de leito - note que $f_{0}$ deve ter um valor constante uma vez que o número de grãos por unidade de volume não muda ao longo do ciclo de filtração - ao passo que $1-f_{d}$ do denominador faz a conversão entre velocidade de aproximação e velocidade intersticial (nos poros). 


\section{Não esfericidade}

Payatakes et al (1981) observaram que a deposição de sólidos durante a filtração ocorre de forma diferente nas camadas e que acarretam diferentes estruturas ao depósito de sólidos no leito filtrante. Segundo Payatakes et al (1981) uma maior compactação, bem como flocos mais coesos ocorrem na camada superior (no caso de filtração descendente) e que, portanto, o desprendimento dependerá da seção do leito filtrante.

Para o caso da filtração ascendente, entretanto, a maior quantidade dos flocos fica retida na camada suporte, e que apesar de sua granulometria maior - que poderia favorecer a dispersão - os flocos tem forte coesão e o depósito mais compacto, indicando a ação hidrodinâmica na formação da estrutura do depósito de sólidos do leito filtrante, o que confirma a observação de Payatakes et al (1981).

Veerapaneni \& Wiesner (1997) consideram que a deposição que ocorre por difusão acarreta um depósito dentrítico (micro-canais) de sólidos cuja característica é a maior compactação do depósito formado.

A não esfericidade é característica fundamental na formação da estrutura do depósito de sólidos e pode ser considerada na formulação com a introdução do fator de forma (S), tanto para os coletores $\left(S_{c}\right)$ como para as partículas $\left(\mathrm{S}_{\mathrm{p}}\right)$ presentes na água.

A esfericidade $(\Psi)$ é definida como sendo a relação entre superfícies: a superfície de uma esfera de igual volume pela superfície do coletor ou partícula. $\mathrm{O}$ fator de forma $(\mathrm{S})$ é definido como sendo a superfície específica, ou seja: $\mathrm{S}=\mathrm{S}_{\mathrm{v}} \mathrm{d}_{\mathrm{eq}} \quad \mathrm{S}=\frac{6}{\xi}$, onde $\mathrm{S}_{\mathrm{V}}$ é a superfície pelo volume do coletor ou partícula e $\mathrm{d}_{\text {eq }}$ é o diâmetro equivalente ao de uma esfera de igual volume. Para uma partícula ou coletor esférico, $S=6$ e $\Psi=1$.

\section{Perda de carga no regime de Forchheimer}

Para Trussell \& Chang (1999), o escoamento em meios porosos, mesmo para número de Reynolds próximos ao valor da unidade, pode contar com linhas de corrente que apresentam alguma influência inercial.

Segundo Ergun (1952), a perda de carga em meios filtrantes granulares é devida, simultaneamente, a perdas viscosas e cinéticas. A equação desenvolvida por Ergun (1952) pode ser aplicada a todos os tipos de escoamentos, fato observado no gráfico da Figura 1.

Note que a equação de Ergun (linha curva cheia) não está apenas definida para as mesmas regióes definas pelas equações de Carman-Kozeny e de Blake-Plummer (linha tracejada e linha pontilhada, respectivamente) mas também para a região entre os domínios destas duas equações.

Pode-se observar que existe um "descolamento" entre a equação de Carman-Kozeny e os dados experimentais, bem como a equação de Ergun, próximo ao valor para o número de Reynolds $\approx 3$. Trussell \& Chang (1999), mostraram que a filtração em meios porosos ocorre, em geral, no Regime de Forchheimer (Escoamento laminar) em um intervalo do número de Reynolds que varia entre 0,5 e 50 .

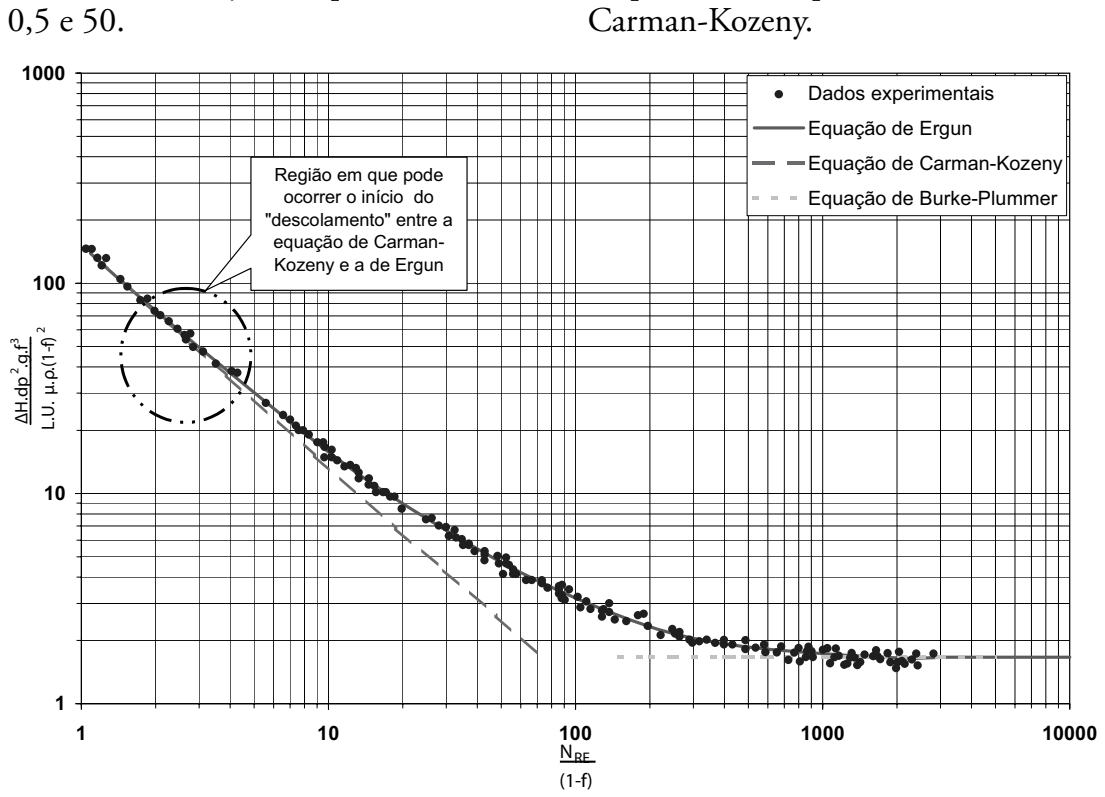

Fonte: Botari (2007)

Figura I - Representação gráfica do comportamento das equações de perda de carga em relação ao número de Reynolds em meios filtrantes

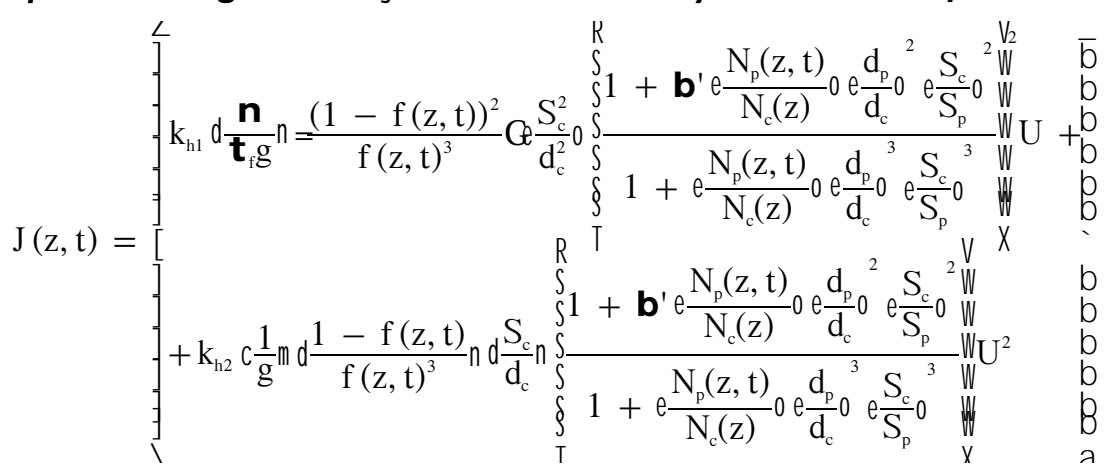
onde: $\mathrm{J}(\mathrm{z}, \mathrm{t})$ é a variação na perda de carga ao longo do tempo e do espaço (m); $\mathrm{p}_{\mathrm{f}}$ densidade do fluido $\left(\mathrm{Kg} \cdot \mathrm{m}^{-1} \cdot \mathrm{s}^{-1}\right)$; g aceleração da gravidade $\left(\mathrm{m} . \mathrm{s}^{-2}\right)$; U viscosidade dinâmica do fluido $\left(\mathrm{kg} \cdot \mathrm{m}^{-1} \mathrm{~s}^{-1}\right) ; \mathrm{k}_{\mathrm{h} 1}$ e $\mathrm{k}_{\mathrm{h} 2}$ são constantes da equação de Ergun (adimensionais; $S_{c}$ e $S_{p}$ são o fator de forma de coletor e da partícula (adimensionais), respectivamente. 


\section{Gradação granulométrica}

Para leitos fluidificados os grãos ou coletores mais finos alinham-se na parte superior ao passo que os maiores ficam um pouco mais abaixo e assim sucessivamente. Esta gradação deve ser considerada na formulação do modelo matemático.

Será utilizada uma interpolação linear entre os coletores (grãos) de diâmetro $d_{10}$ (os 10\% de grãos de diâmetros menores) e os de diâmetro $d_{90}(90 \%$ dos diâmetros restantes) para a gradação dos diâmetros dos grãos coletores. A gradação granulométrica do respectivo valor do diâmetro médio é função da profundidade ("passo" $(\Delta \mathrm{z})$ na resolução das equaçóes do modelo) no leito filtrante $\left(d_{c}(z)\right)$, incrementado de baixo para cima, conforme Equação (15):

$\mathrm{d}_{\mathrm{c}}(\mathrm{z})=\mathrm{d}_{90}-{ }^{\wedge} \mathrm{d}_{90}-\mathrm{d}_{10} h \frac{\mathrm{Z}}{\mathrm{L}}$

onde $L$ é a profundidade do filtro ou da camada (m) em questão.

$\mathrm{O}$ valor do diâmetro do coletor será o valor da média geométrica $-\mathrm{d}_{\mathrm{c}}(\bar{z})$ - entre o valor do diâmetro em $z_{i}$, e $z_{i+1}$, conforme Equação (16) e esquema da Figura 2:

$\mathrm{d}_{\mathrm{c}_{-}} \overline{\mathrm{z}}_{\mathrm{i}} \mathrm{i}=\sqrt{\mathrm{d}_{\mathrm{c}}\left(\mathrm{z}_{\mathrm{i}}\right) \mathrm{d}_{\mathrm{c}_{-} \mathrm{z}_{\mathrm{i}}+\mathrm{i}^{\mathrm{i}}}}$

onde $d_{c}\left(\overline{z_{i}}\right)$ é o diâmetro médio (geométrico) do coletor na posição $i$, entre o espaço $z_{i}$ e $z_{i+1}, d_{c}\left(z_{i}\right)$ é o diâmetro do coletor no espaço $\mathrm{z}_{\mathrm{i}}$ e $\mathrm{d}_{\mathrm{c}}\left(\mathrm{z}_{\mathrm{i}+1}\right)$ é o diâmetro do coletor no espaço $z_{i+1}$, conforme esquema da Figura 2.

\section{Leito multicamadas}

Pode-se considerar as múltiplas camadas de um filtro utilizando-se a variação ou gradação de seus respectivos tamanhos para cada camada. Não se considerará misturas entre camadas do leito de múltiplas camadas.

\section{METODOLOGIA}

\section{Resolução das Equações}

A solução para o conjunto das equações (4), (7), (8), (10), (11), (13) e (14), na forma diferencial e sua integração, é conforme apresentado no esquema da Figura 2.

No início da filtração considera-se o meio filtrante limpo, o que torna a eficiência de remoção bem conhecida, sendo igual a $\eta_{0} \alpha_{0}$. Portanto, a con- centração de partículas no filtro pode também ser determinada $(t=0)$ e é igual a $n_{0}$. A partir de então, no próximo passo, o total de partículas na posição $\mathrm{z}_{\mathrm{i}+1}\left(\mathrm{n}_{\mathrm{i}+1, \mathrm{j}}\right)$ será o resultado da soma das partículas existentes na posição $\mathrm{z}_{\mathrm{i}}\left(\mathrm{n}_{\mathrm{i}, \mathrm{j}}\right)$ mais as partículas retidas na camada $i\left(\eta_{\mathrm{i}, \mathrm{j}}\right)$, para todo $j$, conforme Figura 2.

A diferenciação no tempo $(\Delta \mathrm{t})$ e no espaço $(\Delta z)$ para uma dada camada do leito filtrante, pode ser observada nas equações: $n_{i, j}=n\left(z_{i}, t_{j}\right), \eta_{i, j}=\eta\left(z_{i}, t_{j}\right)$, $N_{i, j}=N\left(z_{i}, t_{j}\right)$

As condições iniciais de cálculo, são as seguintes:

$$
\begin{aligned}
& : \mathrm{N}_{\mathrm{Li}}{ }^{\wedge} \mathrm{z}, 0 \mathrm{~h}=0 ; \mathrm{h}_{\mathrm{i}}{ }^{\wedge} \mathrm{z}, 0 \mathrm{~h}=
\end{aligned}
$$

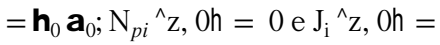

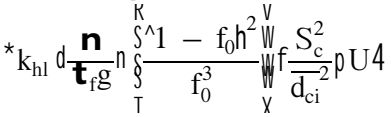

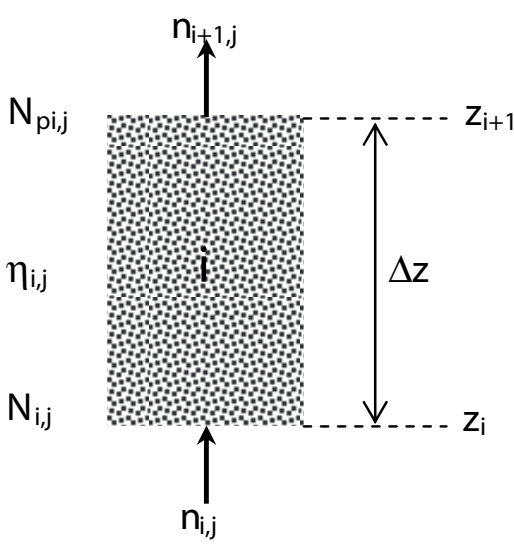

Fonte: Botari, A. (2007)

Figura 2 - Esquema da diferenciação no tempo $t_{j}$ para $a$ camada de material filtrante no espaço $z_{i}$ e $z_{i+1}$

\section{Algoritmo de regressão não-linear para equações multivariáveis}

Para o ajuste dos parâmetros observados na formulação $\left(\eta_{0} \alpha_{0}, \beta \eta_{p} \alpha_{p}\right.$, $\beta_{2}$ e $\beta$ ) e sua compatibilização com as equaçóes do modelo, assim como com os dados experimentais, é indicada a utilização de um algoritmo de regressão não-linear para equações multivariáveis, como é o caso do algoritmo de Marquardt (BSOLVE) baseado no procedimento proposto por Marquardt (1963) e que mais tarde ficou conhecido como método de Levenberg-Marquardt.
Segundo Kuester (1973), a utilização da função-objetivo dos mínimos quadrados permite maior confiabilidade ao método de Levenberg-Marquardt, que tem sua convergência acelerada se comparada ao método de GaussNewton, apesar da possibilidade da adoção de valores iniciais distantes dos valores reais dos parâmetros.

Marquardt (1963) desenvolveu regras em seu algoritmo para a determinação do valor do fator de correção que permitem uma aceleração da resolução, bem como determinar o tamanho do "passo" mais satisfatório para uma rápida convergência para a maioria dos casos de otimização de equações ou conjunto de equações não lineares. $\mathrm{O}$ algoritmo utilizado neste trabalho foi desenvolvido em linguagem FORTRAN para Windows.

\section{Dados experimentais}

A Figura 3a mostra a Instalação Piloto na qual foram obtidos os dados experimentais utilizados para alimentar o modelo. O sistema é composto por dois Filtros Ascendentes de Areia Grossa (FAAG) e um Filtro Rápido Descendente (FRD), conforme Figura 3a.

No desenho esquemático da figura 3b, tem-se: $\mathrm{C} n$ - Pontos de coleta de amostra, onde $n$ é a camada relacionada na Tabela 1. "Coagulada", é o ponto de coleta de água bruta coagulada ou $C_{o} ; \mathrm{P}_{n+1}$ - Pontos de tomadas piezométricas no topo da camada, onde $n$ é a respectiva camada relacionada na Tabela 1 (o índice 1 é relativo à entrada de água bruta coagulada) e $n(1,2 \ldots 6)$ é a camada relacionada na Tabela 1 .

Os pontos de coleta de amostra, bem como os de tomada piezométrica das camadas, doravante mencionados neste trabalho, estarão referidos a este esquema.

Os materiais granulares utilizados no filtro têm as seguintes características gerais: tamanho dos grãos: 1,19 a $3,36 \mathrm{~mm}$, tamanho efetivo $=1,41 \mathrm{~mm}$, coeficiente de desuniformidade $=1,42$ e espessura da camada $=1,4 \mathrm{~m}$. A caracterização detalhada do meio filtrante do filtro ascendente de areia grossa é apresentada na Tabela 1 .

A Tabela 2 mostra as características físicas gerais do fluido e um resumo dos parâmetros das simulaçōes que são apresentadas no item de resultados.

Foram preparados dois tipos de água: a água tipo I e a água tipo II, com turbidez de aproximadamente 100 e $300 \mathrm{uT}$, 
respectivamente. A turbidez fora introduzida através da utilização do material sobrenadante da suspensão de caulinita preparada com água do poço (EESCUSP) e sua posterior homogeneização.

Foram obtidos os valores de turbidez ao longo da carreira de filtração nos pontos de coleta supramencionados com base na leitura em turbidímetro de bancada do tipo Hach, modelo 210 P. Os resultados, entretanto, são apresentados em termos de Sólidos Suspensos Totais (SST), uma vez que foram levantadas curvas de correlação entre turbidez e concentração de SST para diversas faixas de valores de turbidez (i.e. entre 1 e 10; 10 e 100; 100 e 400 ou 100 e 800 uT, este último no caso da água Tipo II), conforme metodologia especificada em Botari (2007).

Foram verificadas três taxas nas carreiras de filtração: $8,33 \mathrm{~cm} \cdot \mathrm{min}^{-1}$ $\left(120 \mathrm{~m}^{3} \cdot \mathrm{m}^{-2} \cdot \mathrm{dia}^{-1}\right), 12,5 \mathrm{~cm} \cdot \mathrm{min}^{-1}(180$ $\left.\mathrm{m}^{3} \cdot \mathrm{m}^{-2} \cdot \mathrm{dia}^{-1}\right)$ e $16,67 \mathrm{~cm} \cdot \mathrm{min}^{-1}(240$ $\left.\mathrm{m}^{3} \cdot \mathrm{m}^{-2} \cdot \mathrm{dia}^{-1}\right)$. O coagulante utilizado foi o sulfato de alumínio e as condições ótimas de dosagem e mistura rápida (tempo e gradiente de velocidade) foram obtidas por meio de ensaios em jarteste e kit de filtros de laboratório de areia (FLA).

\section{RESULTADOS E DISCUSSÃO}

Quando os valores dos parâmetros do modelo estiverem seguidos das letras maiúsculas "SR" (Sem Restrição), tais parâmetros não terão as restriçôes de valores inferiores à unidade (1). A Tabela 3 apresenta um resumo das simulaçōes do ensaio 1 para a camada C1, conforme esquema da Figura $3 b$, ao passo que a Figura 4 mostra os resultados das simulaçóes e os dados experimentais de eficiência de remoção de massa e a de perda de carga.

No caso da camada de filtração C1 (veja esquema da Figura 3b) do ensaio 1, pôde-se observar que a perda de carga para condições de restrição dos coeficientes do modelo de filtração (valores de $\eta_{o} \alpha_{o}, \beta \eta_{p} \alpha, \beta$ e $\beta$ 'inferiores ou iguais à unidade) apresentou boa aderência aos resultados experimentais. Isto pode ser observado visualmente no gráfico da Figura 4 e pelos valores dos coeficientes de correlação não linear $\left(\mathrm{R}^{2}\right)$ da Tabela 3.

No tocante à eficiência de remoção, os valores com restrição mostraram-se razoáveis em vista dos valores experimentais, no caso da camada de filtração $\mathrm{C} 1$, fato corroborado pelo valor do coeficiente de correlação não linear (Tabela 3). É i nteressante observar que no caso da camada C2, do ensaio 2 (gráfico da Figura 5), o mesmo fato não ocorre, tendo-se obtido bom grau de correlação apenas para condiçôes sem restrição tanto para a modelação da perda de carga quanto para a eficiência de remoção de massa (ou partículas) - Tabela 4.

$\mathrm{Na}$ camada de filtração C3 do ensaio 3, a modelação da perdade de carga não apresentou tanta aderência aos dados experimentais como no caso da camada C1 do ensaio 1 (Figura 4 e Tabela 3), mesmo não havendo restrição aos valores dos coeficientes do modelo de filtração. Um aspecto interessante, a ser ressaltado, é o fato de que a remoção das restrições aos coeficientes, por si só, não garante melhora no desempenho do modelo em descrever o fenômeno experimental. O observado na Tabela 3 e na Figura 4, corroboram este fato.

Para a camada de filtração C4 do ensaio 1 (Tabela 6 e Figura 7), observa-se que igualmente, apenas para valores sem restriçóes dos coeficientes do modelo, tanto para a perda de carga quanto para a eficiência de remoção, obteve-se um bom ajuste das curvas dos

Tabela I - Camada suporte e meio filtrante do Filtro Ascendente de Areia Grossa (FAAG)

\begin{tabular}{cccc}
\hline Camada & Subcamada & Tamanho $(\mathrm{mm})$ & Espessura $(\mathrm{cm})$ \\
\hline 6 (topo) & - & 1,19 a 1,41 & 14,0 \\
5 & & 1,41 a 1,68 & 28,0 \\
4 & & 1,68 a 2,00 & 42,0 \\
3 & & 2,00 a 2,38 & 35,0 \\
2 & 1 & 2,38 a 3,36 & 21,0 \\
1 (suporte) & 2 & 12,7 a 19,0 & 7,5 \\
& 3 & 6,4 a 12,7 & 7,5 \\
& 4 & 3,36 a 6,4 & 15,0 \\
& 5 & 6,4 a 12,7 & 7,5 \\
& 6 (fundo) & 12,7 a 19,0 & 7,5 \\
& & 19,0 a 25,4 & 10,0 \\
\hline
\end{tabular}

Fonte: Botari (2007)

Tabela 2 - Quadro das características físicas gerais do fluido e dos parâmetros das simulações

Parâmetros das simulaçóes

$\rho_{f}$ massa específica do fluido $=1000,00\left(\mathrm{~kg} \cdot \mathrm{m}^{-3}\right) ; g$ aceleração da gravidade $=9,81\left(\mathrm{~m} . \mathrm{s}^{-2}\right) ; \mu$ viscosidade dinâmica do fluido que para $T=298 \mathrm{~K}$ é $=0,000894\left(\mathrm{~kg} \cdot \mathrm{m}^{-1} \cdot \mathrm{s}^{-1}\right) ; T$ temperatura absoluta em Kelvin $=298(\mathrm{~K})$ e $f_{d}$ porosidade do depósito $=0,70$ (adimensional). 


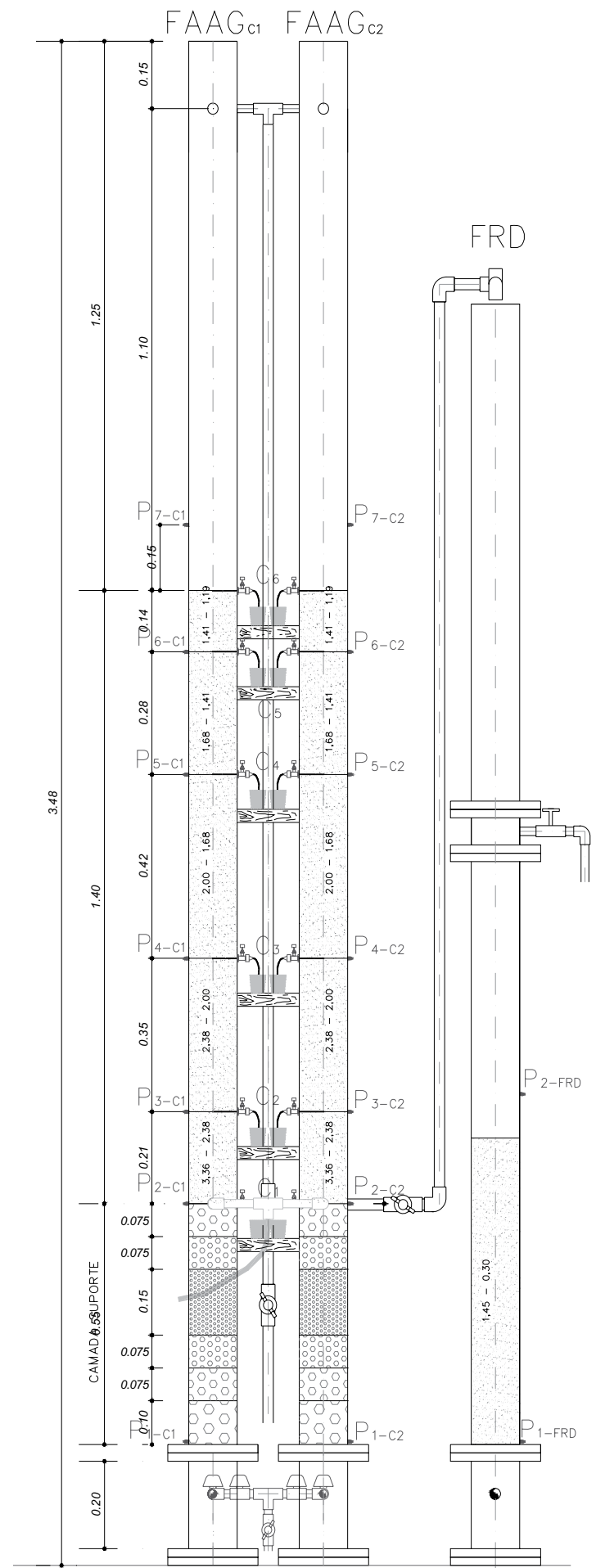

Fonte: adaptado de Di Bernardo, A. (2004). Obs: medidas em metro (m).

Figura 3a - Detalhe da instalação piloto de dupla filtração

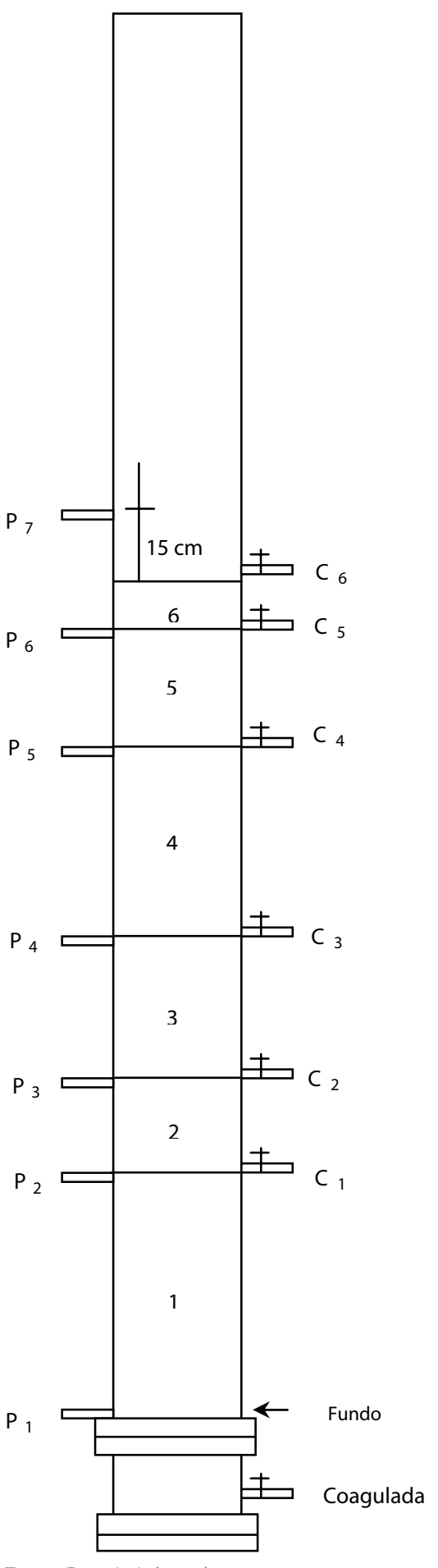

Fonte: Botari, A (2007)

Figura $3 b$ - Desenho esquemático do filtro ascendente da instalação piloto de dupla filtração 
Tabela 3 - Quadro resumo dos dados experimentais e das simulações - ensaio I - camada Cl

\section{Dados da simulaçáo}

Carreira ou ciclo de filtração: ensaio 1; Camada do leito filtrante: C1; Água tipo II

\section{Meio poroso}

Profundidade ou espessura da camada $(L)=0,55 \mathrm{~m}$; Porosidade $(f)=0,40$ (adimensional)

Diâmetro do Coletor $\left(d_{c}\right)$ (máx.) = 25,4 mm; $($ mín $)=3,36 \mathrm{~mm}$; Fator de Forma $(S)=7,5$

\section{Efluente e condiçóes operacionais}

Diâmetro médio da partícula $\left(d_{p}\right)=2,1 \mu \mathrm{m}$; Fator de Forma $(S)=10,25$

Concentração de SST na água de estudo $\left(C_{0}\right)=172,67 \mathrm{mg} \cdot \mathrm{L}^{-1}$; Taxa de Filtração $(U)=12,50 \mathrm{~cm} \cdot \mathrm{min}^{-1}$

Duração da carreira de filtração na modelação $(t)=1230 \mathrm{~min}$

\section{Parâmetros do modelo}

Remoção Inicial $-\eta_{0} \alpha_{0}$ (adimensional) $=3,267.10^{-2}$

Período de Maturação $-\beta \eta_{p} \alpha_{p}($ adimensional $)=3,287.10^{-4}$

Desprendimento $-\beta_{2}\left(s^{-1}\right)=0,3085$

Perda de carga $-\beta^{\prime}$ (adimensional) $=0,9871$

$\mathrm{R}^{2}$ (perda de carga) $=0,9962$

$\mathrm{R}^{2}($ Concentração $)=0,8260$

$\mathrm{R}^{2}$ (média) $=0,9111$

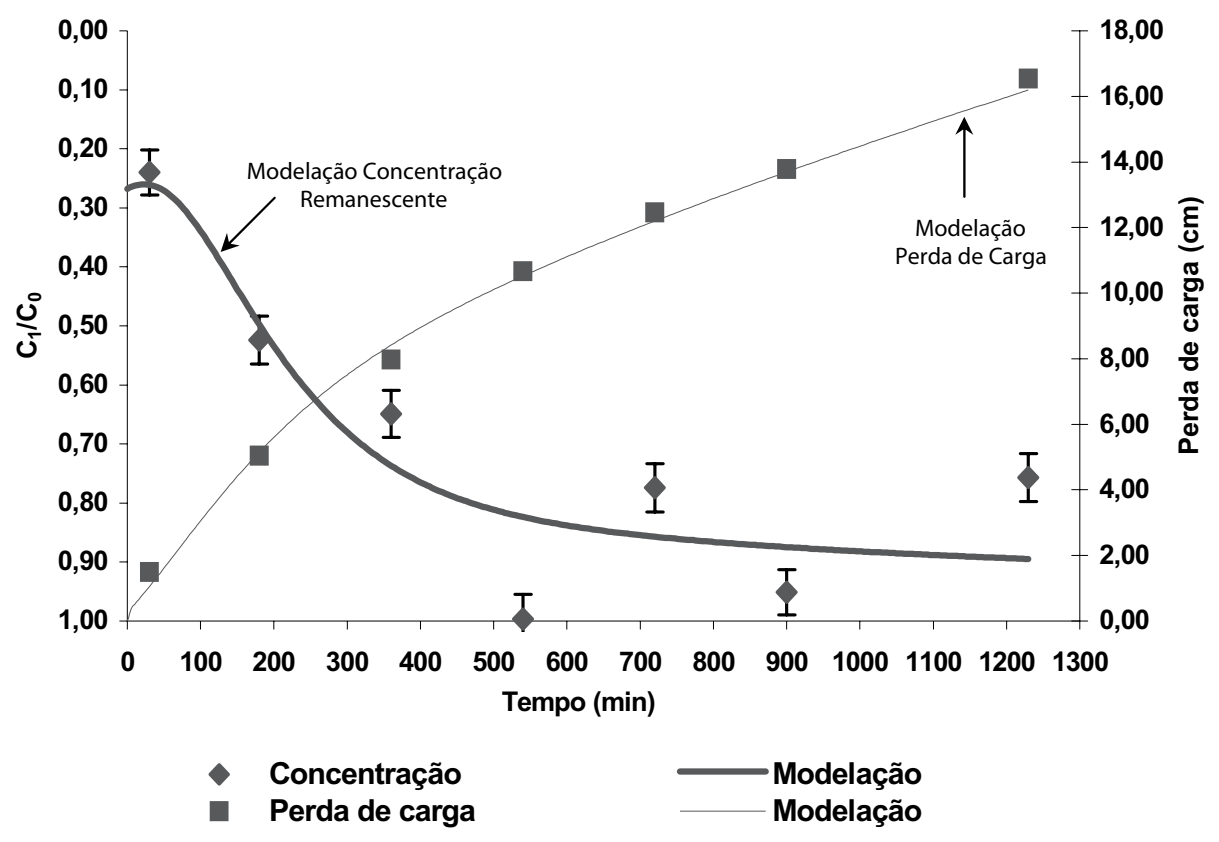

Figura 4 - Perfil da concentração de SST remanescente e da perda de carga ao longo do tempo de filtração - dados experimentais e das simulações - camada CI do ensaio I 
Tabela 4 - Quadro resumo dos dados experimentais e da simulação - ensaio 2 - camada

\section{Dados da simulação}

Carreira ou ciclo de filtração: ensaio 2; Camada do leito filtrante: C2; Água tipo I

\section{Meio poroso}

Profundidade ou espessura da camada $(L)=0,21 \mathrm{~m}$; Porosidade $(f)=0,39$ (adimensional)

Diâmetro do Coletor $(d):(\max )=3,.36 \mathrm{~mm}$; $($ mín. $)=2,38 \mathrm{~mm}$; Fator de Forma $(S)=7,8$

\section{Efluente e condiçóes operacionais}

Diâmetro médio da partícula $\left(d_{p}\right)=2,1 \mu \mathrm{m}$; Fator de Forma $(S)=10,25$

Concentração de SST na água de estudo $\left(C_{0}\right)=57,60 \mathrm{mg}$. $\mathrm{L}^{-1}$; Taxa de Filtração $(U)=12,50 \mathrm{~cm} \cdot \mathrm{min}^{-1}$

Duração do ciclo ou carreira de filtração na modelação $(t)=1860 \mathrm{~min}$

\section{Parâmetros do modelo}

Remoção Inicial $-\eta_{0} \alpha_{0}$ (adimensional) $=2,289.10^{-2}$

Período de Maturação $-\beta \eta_{p} \alpha_{p}$ (adimensional) $=1.10^{-7}(\mathrm{SR})$

Desprendimento $-\beta_{2}\left(s^{-1}\right)=6,416.10^{-2}(S R)$

Perda de carga - $\beta^{\prime}$ (adimensional) $=2,492(\mathrm{SR})$

$\mathrm{R}^{2}$ (perda de carga) $=0,9406(\mathrm{SR})$

$\mathrm{R}^{2}($ Concentração $)=0,9832(\mathrm{SR})$

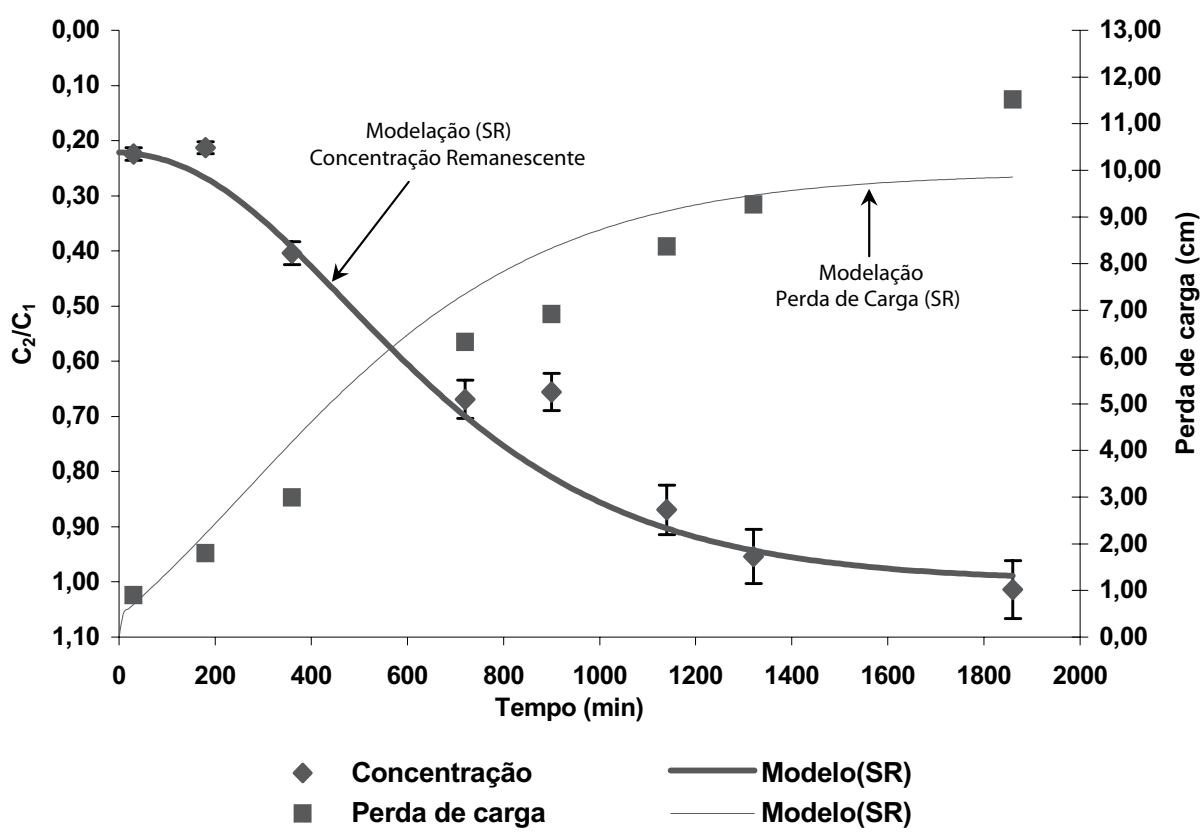

Figura 5 - Perfil da concentração de SST remanescente e da perda de carga ao longo do tempo de filtração - dados experimentais e das simulações - camada C2 do ensaio 2 


\section{Dados da simulaçáo}

Carreira ou ciclo de filtração: ensaio 3; Camada do leito filtrante: C3; Água tipo I

\section{Meio poroso}

Profundidade ou espessura da camada $(L)=0,35 \mathrm{~m}$; Porosidade $(f)=0,39$ (adimensional)

Diâmetro do coletor $(d)$ (máx.) = 2,38 mm; $($ mín $)=2,00 \mathrm{~mm}$; Fator de Forma $(S)=7,7$

\section{Efluente e condiçóes operacionais}

Diâmetro médio da partícula $(\mathrm{dp})=2,1 \mu \mathrm{m}$; Fator de Forma $(S)=10,25$

Concentração de SST na água de estudo $\left(C_{0}\right)=64,53 \mathrm{mg}$. $\mathrm{L}^{-1}$; Taxa de Filtração $(U)=16,67 \mathrm{~cm} \cdot \mathrm{min}^{-1}$

Duração do ciclo ou carreira de filtração na modelação $(t)=1080 \mathrm{~min}$

\section{Parâmetros do modelo}

Remoção inicial $-\eta_{0} \alpha_{0}$ (adimensional) $=1,924.10^{-3}$

Período de maturação $-\beta \eta_{p} \alpha_{p}$ (adimensional) $=0,4186(\mathrm{SR})$

Desprendimento $-\beta_{2}\left(\mathrm{~s}^{-1}\right)=4,933$ (SR)

Perda de carga $-\beta^{\prime}$ (adimensional) $=6,757(\mathrm{SR})$

$\mathrm{R}^{2}$ (perda de carga) $=0,9293(\mathrm{SR})$

$\mathrm{R}^{2}$ (Concentração) $=0,8867(\mathrm{SR})$

$\mathrm{R}^{2}$ (média) = 0,9080 (SR)

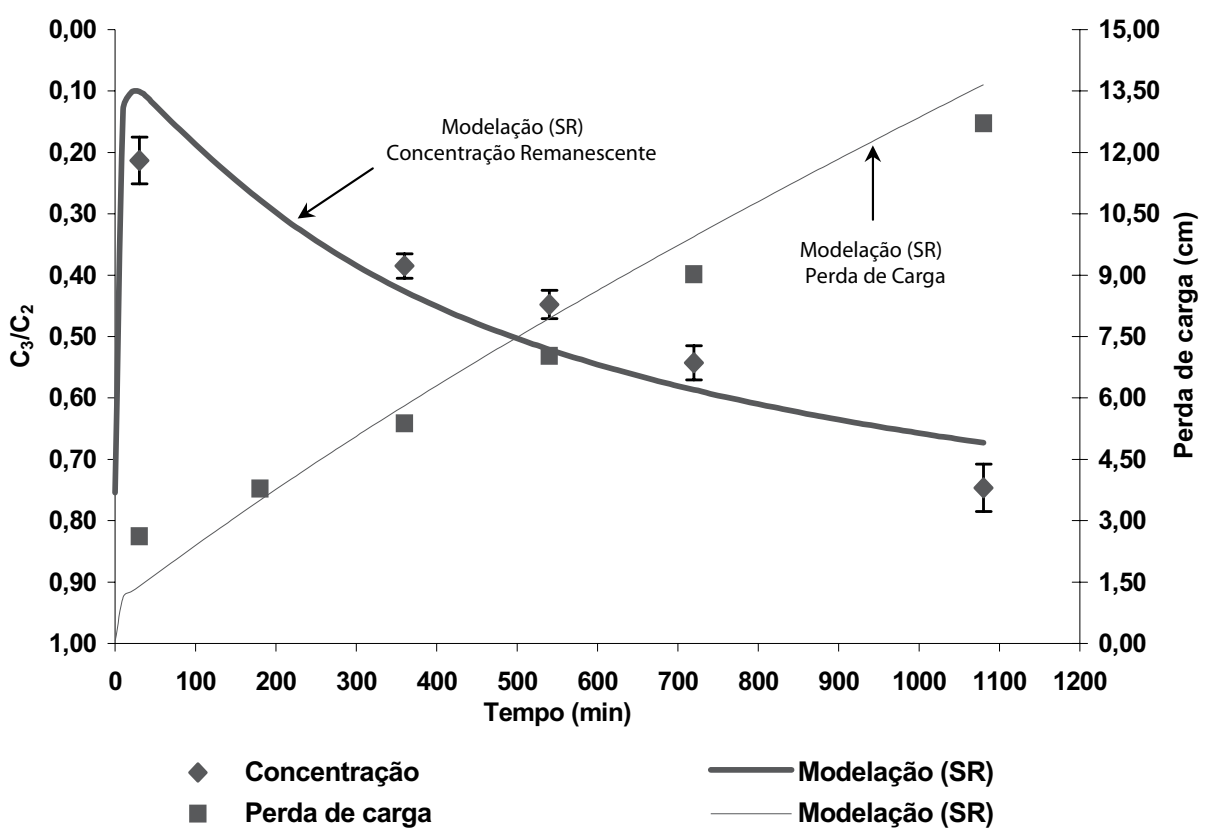

Figura 6 - Perfil da concentração de SST remanescente e da perda de carga ao longo do tempo de filtração - dados experimentais e das simulações - camada C3 do ensaio 3 
Tabela 6 - Quadro resumo dos dados experimentais e das simulações - ensaio I - camada C4

Dados da simulaçáo

Carreira ou ciclo de filtração: ensaio 1; Camada do leito filtrante: C4; Água tipo II

\section{Meio poroso}

Profundidade ou espessura da camada $(L)=0,42(\mathrm{~m})$;Porosidade $(f)=0,39$ [adimensional]

Diâmetro do coletor $(d)$ (máx.) = 2,00 mm; $($ mín) = 1,68 mm; Fator de Forma $(S)=7,6$

\section{Efluente e condiçóes operacionais}

Diâmetro médio da partícula $\left(d_{p}\right)=2,1 \mu \mathrm{m}$; Fator de Forma $(S)=10,25$

Concentração de SST na água de estudo $\left(C_{0}\right)=172,67 \mathrm{mg} \cdot \mathrm{L}^{-1}$; Taxa de Filtração $(U)=12,50 \mathrm{~cm} \cdot \mathrm{min}^{-1}$

Duração do ciclo ou carreira de filtração na modelação $(t)=1230 \mathrm{~min}$

Parâmetros do modelo

$$
\begin{gathered}
\text { Remoção Inicial }-\eta_{o} \alpha_{o}(\text { adimensional })=7,955 \cdot 10^{-3} \\
\text { Período de Maturação }-\beta \eta_{p} \alpha_{p}(\text { adimensional })=1,000 \cdot 10^{-7}(\mathrm{SR}) \\
\text { Desprendimento }-\beta_{2}\left(\mathrm{~s}^{-1}\right)=2,025 \cdot 10^{-2}(\mathrm{SR}) \\
\text { Perda de carga } \left.-\beta^{\prime} \text { (adimensional }\right)=2,529(\mathrm{SR}) \\
\mathrm{R}^{2}(\text { perda de carga })=0,9751(\mathrm{SR}) \\
\mathrm{R}^{2}(\text { Concentração })=0,8674(\mathrm{SR}) \\
\mathrm{R}^{2} \text { (média) }=0,9213(\mathrm{SR})
\end{gathered}
$$

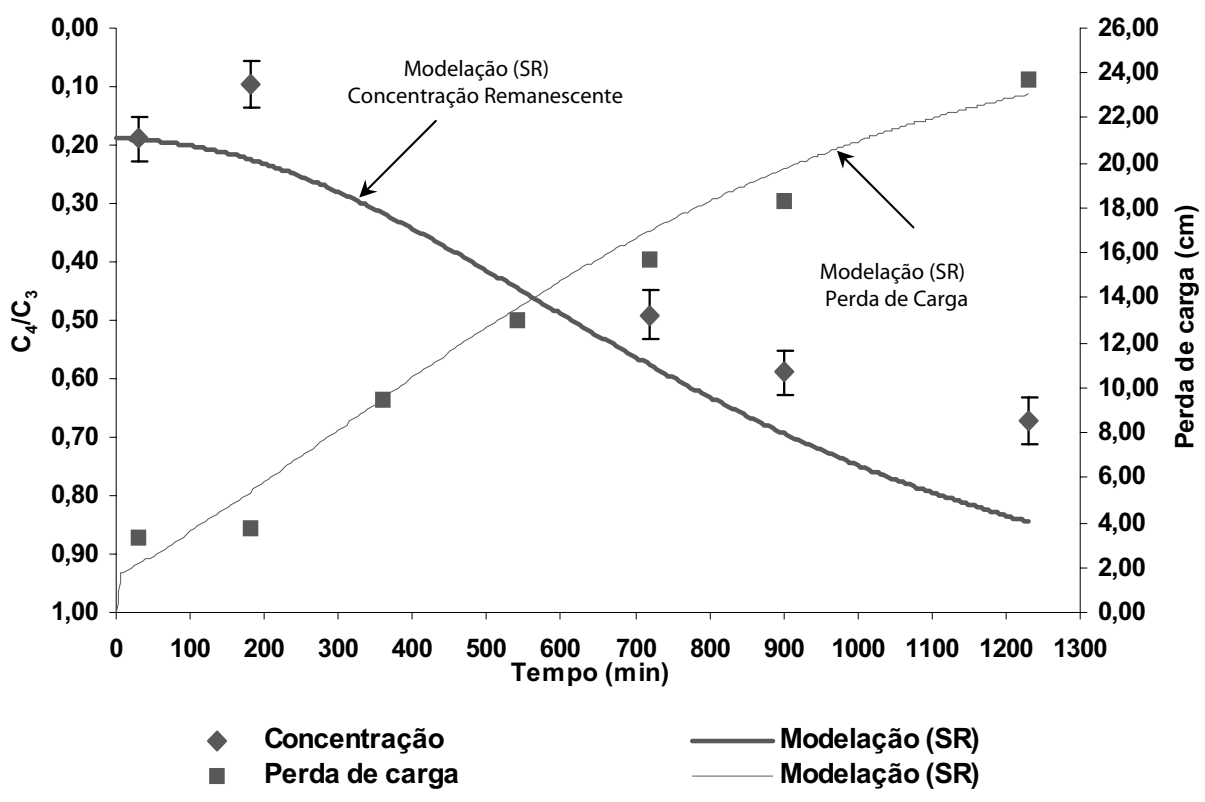

Figura 7 - Perfil da concentração de SST remanescente e da perda de carga ao longo do tempo de filtração - dados experimentais e das simulações - camada C4 do ensaio I 
Entretanto, exigiu-se, muitas vezes, a eliminação da restrição do parâmetro de perda de carga ( $\left.\beta^{\prime}\right)$.

Para o caso da eficiência de remoção de massa, os coeficientes de correlação não linear apresentam menores valores. $\mathrm{O}$ modelo apresenta alguma dificuldade para aderir aos dados experimentais quando a curva necessite de uma conformação tal que inclua acentuados pontos de inflexão ou mudança de declividade (ascendente/descendente), como pode ser visto nas Figuras 5 (para curva de perda de carga) e 7 (para curva de concentração de SST remanescente). Outra limitação do modelo, é a impossibilidade de permitir valor de concentração remanescente superior a 1 .

De forma geral, entretanto, tomando-se os melhores resultados (com ou sem restriçôes aos valores dos coeficientes do modelo) obteve-se uma média de cerca de $93 \%$ de correlação entre a modelação e os dados experimentais para os quatro exemplos apresentados neste trabalho.

Recomendam-se alterações na concepção do modelo de remoção de SST para suprir a deficiência que o modelo utilizado apresenta em descreve-la ao longo do tempo (ou carreira de filtração), em face da existente diferença pronunciada entre os dados modelados e os dados experimentais (mesmo na ausência das restrições aos valores dos coeficientes do referido modelo, como ocorrido nos casos dos gráficos das Figuras 6 e 7).

\section{AGRADECIMENTOS}

Os autores agradecem ao Departamento de Hidráulica e Saneamento da Escola de Engenharia de São Carlos da Universidade de São Paulo - SHS - EESC/USP pelo apoio e instalaçôes e à Coordenação de Aperfeiçoamento de Pessoal de Nível Superior - CAPES pelo apoio financeiro.

\section{REFERÊNCIAS}

BOLLER, M.A.; KAVANAUGH, M.C. Particle Characteristics and Head Loss Increase in Granular Media Filtration. Water Research, n.4, p.1139-49. 1995.

BOTARI, A. Modelação Matemática da Perda de Carga e da Remoção de Turbidez e de Partículas na Filtração Direta Ascendente. São Carlos. 402 p. Tese (Doutorado). Escola de Engenharia de São Carlos, Universidade de São Paulo. 2007.

DARBY, J.L.; ATTANASIO, R.E.; LAWLER, D.F. Filtration of Heterodisperse Suspensions: Modeling of Particle Removal and Head Loss. Water Research, v.26, n.6, p.711-36. 1992.

DI BERNARDO, A. Desempenho de Sistemas de Dupla Filtração no tratamento de água com Turbidez Elevada. São Carlos. 269 p. Tese (Doutorado). Escola de Engenharia de São Carlos, Universidade de São Paulo. 2004.

DI BERNARDO, L. Tratamento de água para abastecimento por Filtração Direta. Projeto PROSAB. Rio de Janeiro: ABES, RiMA. 2003.

ERGUN, S. Fluid Flow Trough Packed Columns. Chemical Engineering Progress, v.42, n.2, p.89-94. 1952

KUESTER, J. L. Optimization Techniques with Fortran. New York: McGraw-Hill. 1973.

LEVEAU, S. Deep Bed Filtration: Analysis and Modeling. Atlanta, U.S.A. 167p. Master of Science Thesis-Georgia Institute of Technology. 2001.
MARQUARDT, D. W. An Algorithm for LeastSquares Estimation of Nonlinear Parameters. Journal of the Society for Industrial and Applied Mathematics, v.11, n.2, p.431-41. 1963.

O'MELIA, C.R.; ALI, W. The Role of Retained Particles in Deep Bed Filtration. Progress in Water Technology, v.10, n.5/6, p.167-82. 1978.

PAYATAKES, A.C.; PARK, H.Y.; PETRIE, J. A Visual Study of Particle Deposition and Reentrainment During Depth Filtration of Hydrosols With Polyelectrolyte. Chemical Engineering Science, v.36, p.1319-35. 1981.

SISSOM, L. E.; PITTS, D. R. Elements of Transport Phenomena. Tokyo: McGraw-Hill Kogakusha Ltd. 1972.

TRUSSELL, R.R.; CHANG, M. Review of Flow Through Porous Media as Applied to Headloss in Water Filters. Journal of Environmental Engineering, p.998-1006, Nov. 1999.

VEERAPANENI, S.; WIESNER, M.R. Deposit Morphology and Head Loss Development in Porous Media. Environmental Science Technology, v.31, n.10, p.2738-44. 1997.

VIGNESWARAN, S.; CHANG, J.S. Mathematical Modeling of the Entire Cycle of Deep Bed Filtration. Water, Air and Soil Pollution, v.29, p.155-64. 1986.

Endereço para correspondência:

Luiz Di Bernardo

Departamento de Hidráulica e

Saneamento

Escola de Engenharia de São

Carlos - USP

Av. Trabalhador São-Carlense,

400

I3566-590 São Carlos - SP - Brasil

Tel.: (16) 3273-9528

Email: bernardo@sc.usp.br 